\title{
Moving Branes with Background Massless and Tachyon Fields in the Compact Spacetime
}

\author{
Zahra Rezaei and Davoud Kamani \\ Physics Department, Amirkabir University of Technology (Tehran Polytechnic) \\ P.O.Box: 15875-4413, Tehran, Iran \\ e-mails: z.rezaei, kamani@aut.ac.ir
}

\begin{abstract}
In this article we shall obtain the boundary state associated with a moving $D p$-brane in the presence of the Kalb-Ramond field $B_{\mu \nu}$, an internal $U(1)$ gauge field $A_{\alpha}$ and a tachyon field, in the compact spacetime. According to this state, properties of the brane and a closed string, with mixed boundary conditions emitted from it, will be obtained. Using this boundary state we calculate the interaction amplitude of two moving $D p_{1}$ and $D p_{2}$-branes with above background fields in a partially compact spacetime. They are parallel or perpendicular to each other. Properties of the interaction amplitude will be analyzed and contribution of the massless states to the interaction will be extracted.
\end{abstract}

PACS numbers: 11.25.-w; 11.25.Mj

Keywords: Moving branes; Boundary state; Background Fields; Interaction. 


\section{Introduction}

Strings are not the only objects of string theory. Since 1995 it has been cleared [1] that the theory includes extended objects which carry charges related to special antisymmetric fields which string theory describes but can not be source of them. These are D-branes which are found to be important in nonperturbative string theories because in strong coupling they become arbitrarily light (lighter than string itself) and dominate the theory in low energies $[2]$.

One of the interesting subjects about the D-branes is interaction between them which is obtainable through two different but equivalent procedures: one loop diagram of open string and tree level diagram of closed string [3]. Since two D-branes interaction can be described by exchanging of closed strings, here we restrict ourselves to the second approach. The state which describes the closed string production from vacuum is called boundary state. Boundary state formalism is a powerful method for studying branes properties and their interactions.

Among achievements in the subject of boundary state formalism is studying the interaction of mixed branes (branes with both Neumann and Dirichlet boundary conditions), moving and angled branes in the presence of background fields such as $\mathrm{U}(1)$ gauge field [4] and antisymmetric field $B_{\mu \nu}[5,6,7,8,9,10,11,12,13]$. Tachyon field also has been added as a background field in some studies $[14,15,16]$.

Since D-branes are not static objects, studying their dynamics is essential to interpret them as physical objects in string theory. Considering velocity for a D-brane which is equal to taking into account the scalar fields from the worldsheet point of view [17], as well as gauge field on the D-brane worldvolume is very instructive to study D-branes dynamics. Besides, progresses in studying open string tachyon field which began mainly by the Sen's works $[15,18,19]$ show that this tachyon field plays an important role on improving our knowledge about D-branes, their instability or stability features, true vacuum of tachyonic string theories and etc [18].

The above facts motivated us to study a system of two moving $\mathrm{D} p_{1}$ and $\mathrm{D} p_{2}$-branes in the presence of the following background: tachyon field, Kalb-Ramond field $B_{\mu \nu}, U(1)$ gauge fields which live in the worldvolumes of the branes, and a partially compacted spacetime on tori. The branes dimensions $p_{1}$ and $p_{2}$ are arbitrary. The relative configurations of the branes are parallel and/or perpendicular. Without fixing the position of the branes, we study both configurations simultaneously. We calculate the boundary state, corresponding to the branes, and then obtain the interaction amplitude between them through exchange of 
closed strings. While the spacetime is allowed to have some compact directions we observe that presence of the tachyon field has some effects on wrapping of the closed string around these directions. In addition, the tachyon field also affects the interaction amplitude of the branes. For example, the behavior of the amplitude for large distance branes has a major deviation from what is expected in the conventional case which will be interpreted.

\section{The boundary state}

We begin with a special sigma-model for the string. This sigma-model action contains the antisymmetric field $B_{\mu \nu}$, tachyon fields, two $U(1)$ gauge fields which live on the worldvolume of the branes and two velocity terms corresponding to the motion of the branes

$$
\begin{aligned}
S= & -\frac{1}{4 \pi \alpha^{\prime}} \int_{\Sigma} d^{2} \sigma\left(\sqrt{-g} g^{a b} G_{\mu \nu} \partial_{a} X^{\mu} \partial_{b} X^{\nu}+\varepsilon^{a b} B_{\mu \nu} \partial_{a} X^{\mu} \partial_{b} X^{\nu}\right) \\
& -\frac{1}{2 \pi \alpha^{\prime}} \int_{(\partial \Sigma)_{1}} d \sigma\left(A_{\alpha_{1}}^{(1)} \partial_{\sigma} X^{\alpha_{1}}+V_{1}^{i_{1}} X^{0} \partial_{\tau} X^{i_{1}}+\left(T^{(1)}+\frac{1}{2} U_{\mu \nu}^{(1)} X^{\mu} X^{\nu}\right)\right) \\
& +\frac{1}{2 \pi \alpha^{\prime}} \int_{(\partial \Sigma)_{2}} d \sigma\left(A_{\alpha_{2}}^{(2)} \partial_{\sigma} X^{\alpha_{2}}+V_{2}^{i_{2}} X^{0} \partial_{\tau} X^{i_{2}}+\left(T^{(2)}+\frac{1}{2} U_{\mu \nu}^{(2)} X^{\mu} X^{\nu}\right)\right),
\end{aligned}
$$

where $\Sigma$ is the worldsheet of the closed string, exchanged between the branes. The boundaries of this worldsheet, i.e. $(\partial \Sigma)_{1}$ and $(\partial \Sigma)_{2}$, are at $\tau=0$ and $\tau=\tau_{0}$, respectively. The $U(1)$ gauge field $A_{\alpha_{2}}^{(2)}$ lives in the $\mathrm{D} p_{2}$-brane, and $V_{2}^{i_{2}}$ is its velocity component along $X^{i_{2}}$ direction. The set $\left\{X^{\alpha_{2}}\right\}$ specifies the directions along the $\mathrm{D} p_{2}$-brane worldvolume and $\left\{X^{i_{2}}\right\}$ shows the directions perpendicular to it. Similar variables with the index " 1 " refer to the D $p_{1}$-brane.

Here we take the background fields $G_{\mu \nu}$ and $B_{\mu \nu}$ to be constant and the profile of the tachyon field is defined as $T^{2}=T_{0}+\frac{1}{2} U_{\mu \nu} X^{\mu} X^{\nu}$ with constant $T_{0}$ and constant symmetric matrix $U_{\mu \nu}$. The advantage of this profile is that the theory will be Gaussian and therefore is exactly solvable [16]. Vanishing the variation of this action with respect to $X^{\mu}(\sigma, \tau)$ gives the equation of motion of $X^{\mu}(\sigma, \tau)$ and boundary state equations of the emitted (absorbed) closed string from (by) the brane. For simplicity, we remove the indices "1" and " 2 " of the variables which refer to the $\mathrm{D} p_{1}$ and $\mathrm{D} p_{2}$-branes. In the interaction of these branes again we shall restore these indices. Therefore, we receive the mixed boundary state equations (i.e. a combination of Dirichlet and Neumann boundary conditions) at $\tau=0$,

$$
\begin{gathered}
{\left[\partial_{\tau}\left(X^{0}-V^{i} X^{i}\right)+\mathcal{F}^{0}{ }_{\alpha} \partial_{\sigma} X^{\alpha}-U^{0}{ }_{\nu} X^{\nu}\right]_{\tau=\tau_{0}}\left|B_{x}, \tau=0\right\rangle=0,} \\
{\left[\partial_{\tau} X^{\bar{\alpha}}+\mathcal{F}^{\bar{\alpha}}{ }_{\beta} \partial_{\sigma} X^{\beta}-U^{\bar{\alpha}}{ }_{\nu} X^{\nu}\right]_{\tau=0}\left|B_{x}, \tau=0\right\rangle=0,} \\
{\left[X^{i}-V^{i} X^{0}-y^{i}\right]_{\tau=0}\left|B_{x}, \tau=0\right\rangle=0}
\end{gathered}
$$


where $\bar{\alpha}$ refers to the spatial directions of the brane (i.e. $\bar{\alpha} \neq 0$ ), $\left\{y^{i}\right\}$ denotes the initial transverse coordinates of the brane, and $\mathcal{F}$ is total field strength

$$
\mathcal{F}_{\alpha \beta}=\partial_{\alpha} A_{\beta}-\partial_{\beta} A_{\alpha}-B_{\alpha \beta}
$$

The first two terms define the field strength of $A_{\alpha}$, which is assumed to be constant. For simplification, we also assumed that the mixed elements of the Kalb-Ramond field to be zero, i.e., $B^{\alpha}{ }_{i}=0$.

To solve these equations we use the general solution of the closed string equation of motion

$$
X^{\mu}(\sigma, \tau)=x^{\mu}+2 \alpha^{\prime} p^{\mu} \tau+2 L^{\mu} \sigma+\frac{i}{2} \sqrt{2 \alpha^{\prime}} \sum_{m \neq 0} \frac{1}{m}\left(\alpha_{m}^{\mu} e^{-2 i m(\tau-\sigma)}+\tilde{\alpha}_{m}^{\mu} e^{-2 i m(\tau+\sigma)}\right) .
$$

In this relation $L^{\mu}$ is zero for non-compact directions and $L^{\mu}=N^{\mu} R^{\mu}$ for compact directions where $N^{\mu}$ is the winding number of the closed string and $R^{\mu}$ is the radius of compactification of the compact direction $X^{\mu}$. Therefore, the boundary state Eqs. (2)-(4) can be written in terms of the oscillators

$$
\begin{aligned}
& {\left[\left(\alpha_{m}^{0}-V^{i} \alpha_{m}^{i}-\mathcal{F}^{0}{ }_{\alpha} \alpha_{m}^{\alpha}-\frac{i}{2 m} U^{0}{ }_{\nu} \alpha_{m}^{\nu}\right)\right.} \\
& \left.+\left(\tilde{\alpha}_{-m}^{0}-V^{i} \tilde{\alpha}_{-m}^{i}+\mathcal{F}^{0}{ }_{\alpha} \tilde{\alpha}_{-m}^{\alpha}+\frac{i}{2 m} U^{0}{ }_{\nu} \tilde{\alpha}_{-m}^{\nu}\right)\right]\left|B_{x}, \tau=0\right\rangle=0 \\
& {\left[\left(\alpha_{m}^{\bar{\alpha}}-\mathcal{F}^{\bar{\alpha}}{ }_{\beta} \alpha_{m}^{\beta}-\frac{i}{2 m} U^{\bar{\alpha}}{ }_{\nu} \alpha_{m}^{\nu}\right)\right.} \\
& \left.+\left(\tilde{\alpha}_{-m}^{\bar{\alpha}}+\mathcal{F}^{\bar{\alpha}}{ }_{\beta} \tilde{\alpha}_{-m}^{\beta}+\frac{i}{2 m} U^{\bar{\alpha}}{ }_{\nu} \tilde{\alpha}_{-m}^{\nu}\right)\right]\left|B_{x}, \tau=0\right\rangle=0 \\
& {\left[\left(\alpha_{m}^{i}-V^{i} \alpha_{m}^{0}\right)-\left(\tilde{\alpha}_{-m}^{i}-V^{i} \tilde{\alpha}_{-m}^{0}\right)\right]\left|B_{x}, \tau=0\right\rangle=0}
\end{aligned}
$$

and zero modes

$$
\begin{gathered}
\left.\left[2 \alpha^{\prime}\left(p^{0}-V^{i} p^{i}\right)+2 \mathcal{F}_{\alpha}^{0} L^{\alpha}-U^{0}{ }_{\nu} x^{\nu}\right)_{\mathrm{op}}\right]\left|B_{x}, \tau=0\right\rangle=0, \\
U^{0}{ }_{\nu} L_{\mathrm{op}}^{\nu}\left|B_{x}, \tau=0\right\rangle=0, \\
\left(2 \alpha^{\prime} p^{\bar{\alpha}}+2 \mathcal{F}^{\bar{\alpha}}{ }_{\beta} L^{\beta}-U^{\bar{\alpha}}{ }_{\nu} x^{\nu}\right)_{\mathrm{op}}\left|B_{x}, \tau=0\right\rangle=0, \\
U^{\bar{\alpha}}{ }_{\nu} L_{\mathrm{op}}^{\nu}\left|B_{x}, \tau=0\right\rangle=0, \\
\left(x^{i}-V^{i} x^{0}-y^{i}\right)_{\mathrm{op}}\left|B_{x}, \tau=0\right\rangle=0, \\
L_{\mathrm{op}}^{i}\left|B_{x}, \tau=0\right\rangle=0 .
\end{gathered}
$$




$$
\left(p^{i}-V^{i} p^{0}\right)_{\mathrm{op}}\left|B_{x}, \tau=0\right\rangle=0 .
$$

where we assumed that the time direction is non-compact, i.e. $L^{0}=0$. The index "op" means that the variables are operator.

From now on we put a restriction on the velocities and consider both $\mathrm{D} p_{1}$ and $\mathrm{D} p_{2}$-branes to move along the common direction $X^{i_{0}}$, which is perpendicular to both of them. Thus, the Eqs. (10), (12) and (16), lead to

$$
\begin{aligned}
& p^{0}=\frac{\gamma^{2}}{\alpha^{\prime}}\left(\frac{1}{2} U_{\mu}^{0} x^{\mu}-\mathcal{F}^{0}{ }_{\bar{\beta}} L^{\bar{\beta}}\right), \\
& p^{\bar{\alpha}}=\frac{1}{\alpha^{\prime}}\left(\frac{1}{2} U^{\bar{\alpha}}{ }_{\mu} x^{\mu}-\mathcal{F}^{\bar{\alpha}}{ }_{\bar{\beta}} L^{\bar{\beta}}\right), \\
& p^{i_{0}}=\frac{V \gamma^{2}}{\alpha^{\prime}}\left(\frac{1}{2} U_{\mu}^{0} x^{\mu}-\mathcal{F}^{0}{ }_{\bar{\beta}} L^{\bar{\beta}}\right),
\end{aligned}
$$

where $\gamma=1 / \sqrt{1-V^{2}}$. For the compact direction $X^{\mu}$ we also have $p^{\mu}=M^{\mu} / R^{\mu}$ where $M^{\mu}$ is the momentum number of the closed string. These equations imply that the nonzero momentum components (momentum numbers) of closed string depend on its winding numbers around the wrapped directions of the brane, and its center of mass position. The former is due to the massless background fields, while the latter is the effect of the tachyon field.

Combining the Eqs. (11), (13) and (15) leads to

$$
U^{\alpha}{ }_{\bar{\beta}} L_{\mathrm{op}}^{\bar{\beta}}\left|B_{x}, \tau=0\right\rangle=0 .
$$

If the $p \times p$ square sub-matrix $U^{\bar{\alpha}}{ }_{\bar{\beta}}$ is invertible we obtain

$$
L_{\mathrm{op}}^{\bar{\alpha}}\left|B_{x}, \tau=0\right\rangle=0 .
$$

Thus, the background tachyon field prevents the wrapping of the closed string around the compact directions of the brane. The Eqs. (15) and (19) imply that the closed string can not wrap around any compact direction of the spacetime. Putting aside the invertibility of the sub-matrix $U^{\bar{\alpha}}{ }_{\bar{\beta}}$, the closed string can wind around the compact directions of the brane. However, we assume $U^{\bar{\alpha}}{ }_{\bar{\beta}}$ to be invertible.

Now we solve the boundary state equations to obtain the boundary state. By using the coherent state method [20], the Eqs. (7)-(9) give the oscillating part of the boundary state as in the following

$$
\left|B_{\mathrm{osc}}, \tau=0\right\rangle=\prod_{n=1}^{\infty}\left[\operatorname{det} M_{(n)}\right]^{-1} \exp \left[-\sum_{m=1}^{\infty}\left(\frac{1}{m} \alpha_{-m}^{\mu} \mathcal{S}_{(m) \mu \nu} \widetilde{\alpha}_{-m}^{\nu}\right)\right]|0\rangle,
$$

where

$$
\left\{\begin{array}{l}
\mathcal{S}_{(m)}=S_{(m)}+\left(\left(S_{(-m)}\right)^{-1}\right)^{T} \\
S_{(m)}=M_{(m)}^{-1} N_{(m)}
\end{array}\right.
$$


Since $S_{(m)}$ is mode dependent and generally is not orthogonal, the matrix $\left(\left(S_{(-m)}\right)^{-1}\right)^{T}$ is appeared here. The matrices $M_{(m)}$ and $N_{(m)}$, which depend on $\mathcal{F}, V, B$ and $U$, are defined by

$$
M_{(m) \nu}^{\mu}=\Omega^{\mu}{ }_{\nu}-\frac{i}{2 m} U_{\nu}^{\alpha} \delta_{\alpha}^{\mu}
$$

where

$$
\left\{\begin{array}{l}
\Omega^{0}{ }_{\mu}=\delta_{\mu}^{0}-V \delta^{i_{0}}{ }_{\mu}-\mathcal{F}^{0}{ }_{\alpha} \delta^{\alpha}{ }_{\mu} \\
\Omega^{\bar{\alpha}}{ }_{\mu}=\delta^{\bar{\alpha}}{ }_{\mu}-\mathcal{F}^{\bar{\alpha}}{ }_{\beta} \delta^{\beta}{ }_{\mu} \\
\Omega^{i}{ }_{\mu}=\delta^{i}{ }_{\mu}-V \delta^{i}{ }_{i_{0}} \delta^{0}{ }_{\mu}
\end{array}\right.
$$

and

$$
\left\{\begin{array}{l}
N_{(m) \mu}^{0}=\delta_{\mu}^{0}-V \delta^{i_{0}}{ }_{\mu}+\mathcal{F}^{0}{ }_{\alpha} \delta^{\alpha}{ }_{\mu}+\frac{i}{2 m} U_{\mu}^{0} \\
N_{(m) \mu}^{\bar{\alpha}}=\delta^{\bar{\alpha}}{ }_{\mu}+\mathcal{F}^{\bar{\alpha}}{ }_{\beta} \delta^{\beta}{ }_{\mu}+\frac{i}{2 m} U_{\mu}^{\bar{\alpha}} \\
N_{(m) \mu}^{i}=-\delta^{i}{ }_{\mu}+V \delta^{i}{ }_{i_{0}} \delta^{0}{ }_{\mu}
\end{array}\right.
$$

where $V^{i_{0}} \equiv V$. The infinite factor in $(20)$ can be regularized as

$$
\prod_{n=1}^{\infty}\left[\operatorname{det} M_{(n)}\right]^{-1}=\sqrt{\operatorname{det} \Omega} \operatorname{det} \Gamma\left(\frac{U}{2 i \Omega}+1\right) .
$$

It is seen that if $U=0$ then (25) will be the familiar DBI Lagrangian.

Solving the zero mode equations (10)-(16) by considering $x^{\mu}$ and $p^{\mu}$ as quantum mechanical operators and using their commutation relations, we receive the state

$$
\begin{aligned}
\left|B_{x}, \tau=0\right\rangle^{(0)}=\quad & \frac{T_{p}}{2} \int_{-\infty}^{\infty} \prod_{\mu} d p^{\mu}\left\{\operatorname { e x p } \left[-i \alpha^{\prime}\left(U^{00}-U^{0 i_{0}} V\right)^{-1}\left(P^{0}\right)^{2}\right.\right. \\
& \left.-2 i \alpha^{\prime} \sum_{\bar{\beta}}\left(\frac{\sum_{\bar{\alpha}}\left[\left(1-\frac{1}{2} \delta_{\bar{\alpha} \bar{\beta}}\right) U^{\bar{\beta} \bar{\alpha}} P^{\bar{\alpha}}\right]}{\sum_{\bar{\gamma}}\left(U^{\bar{\beta}} \bar{\gamma} U^{\bar{\beta}} \bar{\gamma}\right)} P^{\bar{\beta}}\right)\right] \\
& \times \delta\left(x^{i_{0}}-V x^{0}-y^{i_{0}}\right) \prod_{j \neq i_{0}} \delta\left(x^{j}-y^{j}\right) \\
& \left.\times \prod_{\alpha}\left|p_{L}^{\alpha}=p_{R}^{\alpha}\right\rangle \prod_{j \neq i_{0}}\left|p_{L}^{j}=p_{R}^{j}=0\right\rangle\left|p_{L}^{i_{0}}=p_{R}^{i_{0}}=\frac{1}{2} V p^{0}\right\rangle\right\},
\end{aligned}
$$

where $P^{\alpha}=p^{\alpha}-V p^{i_{0}} \delta^{\alpha}{ }_{0}$ and $\bar{\alpha}, \bar{\beta}$ and $\bar{\gamma}$ take their values from the spatial directions of the brane. The index $j$ indicates the directions perpendicular to the brane except $i_{0}$. Since the tachyon field prevents the closed string from wrapping around the compact directions, left and right-components of the momentum are equal. This implies that closed string has zero winding numbers, and its momentum components are not discrete. Therefore, in this state there are integrals over them. 


\section{Interaction of the branes}

\subsection{The amplitude}

For calculating the interaction amplitude we need the total boundary state. What was acquired in (20) and (26) is the matter part of it. We should also take into account the boundary state associated with the conformal ghosts. This is due to the fact that we are working in the covariant formalism. So the total boundary state which will be used to calculate the amplitude, is

$$
|B, \tau=0\rangle=\left|B_{\mathrm{osc}}, \tau=0\right\rangle\left|B_{x}, \tau=0\right\rangle^{(0)}\left|B_{\mathrm{gh}}, \tau=0\right\rangle,
$$

where the ghost part is

$$
\left|B_{\mathrm{gh}}, \tau=0\right\rangle=\exp \left[\sum_{m=1}^{\infty}\left(c_{-m} \widetilde{b}_{-m}-b_{-m} \widetilde{c}_{-m}\right)\right] \frac{c_{0}+\widetilde{c_{0}}}{2}|q=1\rangle|\widetilde{q}=1\rangle .
$$

Now we proceed to calculate the interaction amplitude between the $\mathrm{D} p_{1}$-brane and $\mathrm{D} p_{2^{-}}$ brane through closed string exchanging between them. For this purpose we need closed string propagator which is given by $D=2 \alpha^{\prime} \int_{0}^{\infty} d t e^{-t H}$ where $H$ is the closed string Hamiltonian. Overlap of two boundary states, corresponding to the branes, via this propagator defines the interaction amplitude, i.e. $\mathcal{A}=\left\langle B_{1}|D| B_{2}\right\rangle$. Before calculating the interaction amplitude there are some conventions for indices. The set $\{\bar{i}\}$ shows directions perpendicular to both branes except $i_{0},\{\bar{u}\}$ is for the directions along both branes except $0,\left\{\alpha_{1}^{\prime}\right\}$ is used for the directions along the $\mathrm{D} p_{1}$-brane and perpendicular to the $\mathrm{D} p_{2}$-brane, and $\left\{\alpha_{2}^{\prime}\right\}$ indicates the directions along the $\mathrm{D} p_{2}$-brane and perpendicular to the $\mathrm{D} p_{1}$-brane. Therefore, we have the relations

$$
\begin{aligned}
& \left\{\alpha_{1}\right\}=\{\bar{u}\} \cup\left\{\alpha_{1}^{\prime}\right\} \cup\{0\}, \\
& \left\{\alpha_{2}\right\}=\{\bar{u}\} \cup\left\{\alpha_{2}^{\prime}\right\} \cup\{0\}, \\
& \left\{i_{1}\right\}=\{\bar{i}\} \cup\left\{\alpha_{2}^{\prime}\right\} \cup\left\{i_{0}\right\}, \\
& \left\{i_{2}\right\}=\{\bar{i}\} \cup\left\{\alpha_{1}^{\prime}\right\} \cup\left\{i_{0}\right\}, \\
& \{\mu\}=\left\{\alpha_{1}\right\} \cup\left\{i_{1}\right\}=\left\{\alpha_{2}\right\} \cup\left\{i_{2}\right\} .
\end{aligned}
$$

Since the position of the branes are specified by the running indices $\left\{\bar{\alpha}_{1}\right\}$ and $\left\{\bar{\alpha}_{2}\right\}$, the $\mathrm{D} p_{1}$ and $\mathrm{D} p_{2}$-brane can be parallel or perpendicular to each other.

After a long calculation the following interaction amplitude is acquired

$$
\mathcal{A}=\frac{\alpha^{\prime} V_{\bar{u}}}{4(2 \pi)^{d_{\bar{i}}}} \frac{T_{p_{1}} T_{p_{2}}}{\left|V_{1}-V_{2}\right|} \prod_{m=1}^{\infty}\left(\operatorname{det}\left[M_{(m) 1} M_{(m) 2}\right]\right)^{-1}
$$




$$
\begin{aligned}
& \times \int_{0}^{\infty} d t\left\{e^{(d-2) t / 6} \prod_{m=1}^{\infty}\left(\left[\operatorname{det}\left(1-\mathcal{S}_{(m) 1} \mathcal{S}_{(m) 2}^{T} e^{-4 m t}\right)\right]^{-1}\left(1-e^{-4 m t}\right)^{2}\right)\right. \\
& \times\left(\sqrt{\frac{\pi}{\alpha^{\prime} t}}\right)^{d_{\bar{i}_{n}}} \exp \left[-\frac{1}{4 \alpha^{\prime} t} \sum_{\bar{i}_{n}}\left(y_{1}^{\bar{i}_{n}}-y_{2}{ }^{\bar{i}_{n}}\right)^{2}\right] \prod_{\bar{i}_{c}} \Theta_{3}\left(\frac{y_{1}^{\bar{i}_{c}}-y_{2}^{\bar{i}_{c}}}{2 \pi R_{\bar{i}_{c}}} \mid \frac{i \alpha^{\prime} t}{\pi\left(R_{\bar{i}_{c}}\right)^{2}}\right) \\
& \times \int_{-\infty}^{+\infty} \prod_{\alpha_{1}^{\prime}} d p_{1}{ }^{\alpha_{1}} \prod_{\alpha_{2}^{\prime}} d p_{2}{ }^{\alpha_{2}}\left[\exp \left(-\alpha^{\prime} t\left(f^{(+)} f^{(-)}+p_{1}{ }^{\alpha_{1}^{\prime}} p_{1}{ }^{\alpha_{1}^{\prime}}+p_{2}{ }^{\alpha_{2}^{\prime}} p_{2}{ }^{\alpha_{2}^{\prime}}+p_{1}{ }^{\bar{u}} p_{2}{ }^{\bar{u}}\right)\right)\right. \\
& \times \exp \left(i \Phi(12) y_{2}{ }^{i_{0}}-i \Phi(21) y_{1}{ }^{i_{0}}+2 i y_{1}{ }^{\alpha^{\prime}}{ }^{2} p_{2}{ }^{\alpha^{\prime}}{ }_{2}-2 i y_{2}{ }^{\alpha^{\prime}}{ }_{1} p_{1}{ }^{\alpha^{\prime}}{ }_{1}\right) \\
& \left.\left.\times \exp \left(\pi^{0^{T}} Q \pi^{0}+E^{T} \pi^{0}+\pi_{1}{ }^{T} G_{1} \pi_{1}+\pi_{2}{ }^{T} G_{2} \pi_{2}\right)\right]\right\}
\end{aligned}
$$

where $V_{\bar{u}}$ is the common worldvolume. $\bar{i}_{c}$ and $\bar{i}_{n}$ indicate the compact and non-compact parts of $X^{\bar{i}}$, with $d_{\bar{i}_{n}}=\operatorname{dim}\left\{X^{\bar{i}_{n}}\right\}$ and $d_{\bar{i}}=\operatorname{dim}\left\{X^{\bar{i}}\right\}$. The directions $X^{\bar{i}_{c}}$ and $X^{\bar{i}_{n}}$ are perpendicular to both branes. In addition, $R_{\bar{i}_{c}}$ is the radius of compactification and $d$ is the spacetime dimension. We also defined $\pi^{0}=\left(\begin{array}{c}p_{1}^{0} \\ p_{2}^{0}\end{array}\right), \pi_{1}=\left(\begin{array}{c}p_{1}^{\alpha_{1}^{\prime}} \\ p_{1}^{\bar{u}}\end{array}\right)$ and $\pi_{2}=\left(\begin{array}{c}p_{2}^{\alpha_{2}^{\prime}} \\ p_{2}^{\bar{u}}\end{array}\right)$ in which $\pi_{1}$ and $\pi_{2}$ have $\left(d_{\alpha_{1}^{\prime}}+d_{\bar{u}}\right)$ and $\left(d_{\alpha_{2}^{\prime}}+d_{\bar{u}}\right)$ elements, respectively. The notations $\Phi(12)$ and $f^{(+)}$are defined by

$$
\begin{gathered}
\Phi(12)=\frac{1}{V_{2}-V_{1}}\left[\left(1+V_{1} V_{2}\right) p_{2}^{0}-\left(1+V_{1}^{2}\right) p_{1}^{0}\right] \\
f^{(+)}=\frac{1}{\left|V_{2}-V_{1}\right|}\left[\left(1+V_{1}\right)\left(1+V_{2}^{2}\right) p_{2}^{0}-\left(1+V_{2}\right)\left(1+V_{1}^{2}\right) p_{1}^{0}\right] .
\end{gathered}
$$

By exchanging $2 \leftrightarrow 1$ in (31) we receive $\Phi(21)$, and by changing $V_{1} \longrightarrow-V_{1}$ and $V_{2} \longrightarrow-V_{2}$ in $(32), f^{(-)}$can be obtained. The matrices $Q, G_{1}$ and $G_{2}$ and the doublet $E=\left(\begin{array}{c}E_{1} \\ E_{2}\end{array}\right)$, are defined through their elements as in the following

$$
\begin{aligned}
& \left\{\begin{array}{l}
Q_{11}=\frac{\alpha^{\prime} t}{\left(V_{2}-V_{1}\right)^{2}}\left(1+V_{1}^{2}\right)\left(1-V_{2}^{2}\right)+2 i \alpha^{\prime}\left(U_{1}^{00}-U_{1}^{0, i_{0}} V_{1}\right)^{-1}\left(1-V_{2}^{2}\right)^{2} \\
Q_{22}=\frac{\alpha^{\prime} t}{\left(V_{2}-V_{1}\right)^{2}}\left(1+V_{2}^{2}\right)\left(1-V_{1}^{2}\right)-2 i \alpha^{\prime}\left(U_{2}^{00}-U_{2}^{0, i_{0}} V_{2}\right)^{-1}\left(1-V_{1}^{2}\right)^{2} \\
Q_{12}=Q_{21}=\frac{\alpha^{\prime} t}{\left(V_{2}-V_{1}\right)^{2}}\left(1+V_{1}^{2}\right)\left(1+V_{2}^{2}\right)\left(1-V_{1} V_{2}\right)
\end{array}\right. \\
& \left\{\begin{array}{l}
E_{1}=\frac{i}{V_{2}-V_{1}}\left[y_{2}{ }^{i_{0}}\left(1+V_{1}^{2}\right)^{2}-y_{1}{ }^{i_{0}}\left(1+V_{1} V_{2}\right)\right] \\
E_{2}=\frac{i}{V_{2}-V_{1}}\left[y_{1}{ }^{i_{0}}\left(1+V_{2}^{2}\right)^{2}-y_{2}{ }^{i_{0}}\left(1+V_{1} V_{2}\right)\right]
\end{array}\right.
\end{aligned}
$$




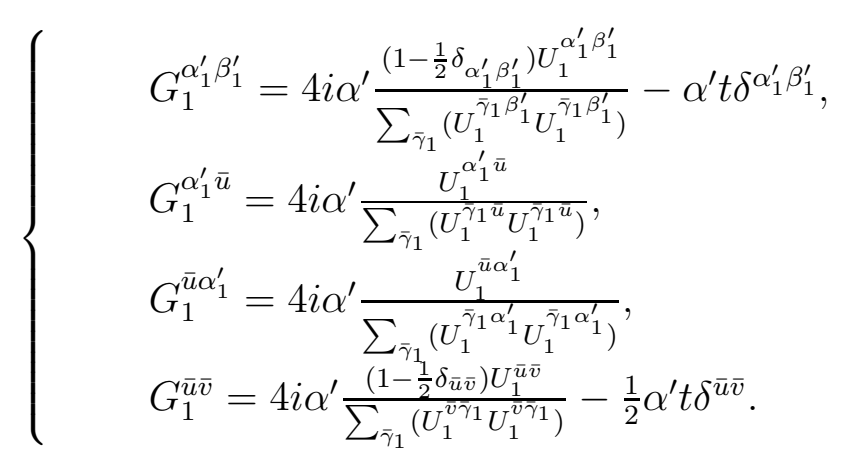

With the exchange $1 \longleftrightarrow 2$ and $i \rightarrow-i$ in the elements of $G_{1}$ we receive the elements of $G_{2}$. For parallel D-branes with the same dimension those terms which contain $\alpha_{1}^{\prime}$ and $\alpha_{2}^{\prime}$ disappear. The effects of compactification are in the product of the $\Theta_{3}$-functions. Therefore, the amplitude in the non-compact spacetime can be obtained as follows: remove the $\Theta_{3^{-}}$ functions, change $\bar{i}_{n} \rightarrow \bar{i}$ and $d_{\bar{i}_{n}} \rightarrow d_{\bar{i}}$.

We can make the amplitude simpler by performing the integration over momenta. After introducing the regularization (25) it finds the feature

$$
\begin{aligned}
\mathcal{A}=\quad & \frac{\alpha^{\prime} V_{\bar{u}}}{4(2 \pi)^{d_{\bar{i}}}} \frac{T_{p_{1}} T_{p_{2}}}{\left|V_{1}-V_{2}\right|} \sqrt{\operatorname{det}\left(\Omega_{1} \Omega_{2}\right)} \operatorname{det}\left[\Gamma\left(\frac{U_{1}}{2 i \Omega_{1}}+1\right) \Gamma\left(\frac{U_{2}}{2 i \Omega_{2}}+1\right)\right] \\
& \times \int_{0}^{\infty} d t\left\{e^{(d-2) t / 6} \prod_{m=1}^{\infty}\left(\left[\operatorname{det}\left(1-\mathcal{S}_{(m) 1} \mathcal{S}_{(m) 2}^{T} e^{-4 m t}\right)\right]^{-1}\left(1-e^{-4 m t}\right)^{2}\right)\right. \\
& \times\left(\sqrt{\frac{\pi}{\alpha^{\prime} t}}\right)^{d_{\bar{i}_{n}}} \exp \left[-\frac{1}{4 \alpha^{\prime} t} \sum_{\bar{i}_{n}}\left(y_{1}^{\bar{i}_{n}}-y_{2}^{\bar{i}_{n}}\right)^{2}\right] \prod_{\bar{i}_{c}} \Theta_{3}\left(\frac{y_{1}^{\bar{i}_{c}}-y_{2}^{\bar{i}_{c}}}{2 \pi R_{\bar{i}_{c}}} \mid \frac{i \alpha^{\prime} t}{\pi\left(R_{\bar{i}_{c}}\right)^{2}}\right) \\
& \times \frac{1}{\sqrt{\operatorname{det} Q \operatorname{det} G_{1} \operatorname{det} G_{2}}} \\
& \left.\times \exp \left[-\frac{1}{4}\left(E^{T} Q^{-1} E+\sum_{\alpha_{1}^{\prime}, \beta_{1}^{\prime}}\left(y_{2}^{\alpha_{1}^{\prime}} y_{2}^{\beta_{1}^{\prime}}\left(G_{1}^{-1}\right)_{\alpha_{1}^{\prime} \beta_{1}^{\prime}}\right)+\sum_{\alpha_{2}^{\prime}, \beta_{2}^{\prime}}\left(y_{1}^{\alpha_{2}^{\prime}} y_{1}^{\beta_{2}^{\prime}}\left(G_{2}^{-1}\right)_{\alpha_{2}^{\prime} \beta_{2}^{\prime}}\right)\right)\right]\right\} .(36)
\end{aligned}
$$

The tachyon, Kalb-Ramond and gauge fields are collected in the matrices $\Omega_{1}, \Omega_{2}, \mathcal{S}_{1}, \mathcal{S}_{2}, Q$, $G_{1}, G_{2}$, and the doublet $E$. The amplitude is symmetric under the exchange of $1 \longleftrightarrow 2$, as expected.

The constant factors behind the integral show the strength of the interaction. The second line in (36) reflects the portion of oscillators and conformal ghosts in interaction. The exponential factor in the third line is a damping factor with respect to the distance of the branes. If all directions $\left\{X^{\bar{i}}\right\}$ are compact then $d_{\bar{i}_{n}}=0$ and this exponential factor disappears. Similarly, if they are non-compact then the $\Theta_{3}$-factor will be eliminated. 


\subsection{Behavior of the interaction amplitude for large distances}

In any interaction theory one should verify the large distances behavior of the amplitude. This gives long-range force of the theory. In our case it is related to the contribution of the closed string tachyon and massless states to the interaction. Now our aim is to verify this statement for our system which contains a special tachyon field. In other words, we intend to study the effect of the background fields on the interaction amplitude after long times. For this purpose we should perform the limit, $\lim _{t \rightarrow \infty} \mathcal{A}$. Since the matrices $Q, G_{1}$ and $G_{2}$ are functions of time, for $d=26$ there is the following limit

$$
\begin{aligned}
& \lim _{t \rightarrow \infty}\left\{e^{4 t} \prod_{m=1}^{\infty}\left(\left[\operatorname{det}\left(1-\mathcal{S}_{(m) 1} \mathcal{S}_{(m) 2}^{T} e^{-4 m t}\right)\right]^{-1}\left(1-e^{-4 m t}\right)^{2}\right)\right. \\
& \left.\times \frac{1}{\sqrt{\operatorname{det} Q \operatorname{det} G_{1} \operatorname{det} G_{2}}} \exp \left(-\frac{1}{4} E^{T} Q^{-1} E\right)\right\} \\
& \left.=\frac{i 2^{d_{\bar{u}}+1 / 2}(-1)^{\left(p_{1}+p_{2}\right) / 2}}{\alpha^{\prime\left(p_{1}+p_{2}\right) / 2}} \frac{\left|V_{1}-V_{2}\right|}{\left(1+V_{1}^{2}\right)\left(1+V_{2}^{2}\right)} \lim _{t \rightarrow \infty}\left\{\frac{e^{4 t}}{t^{1+\left(p_{1}+p_{2}\right) / 2}}+\frac{\operatorname{Tr}\left(\mathcal{S}_{(1) 1} \mathcal{S}_{(1) 2}^{T}\right)-2}{t^{1+\left(p_{1}+p_{2}\right) / 2}}\right\} 37\right)
\end{aligned}
$$

Substituting the limit (37) into the amplitude (36), the massless states and tachyon contributions to the interaction amplitude for $d=26$ become

$$
\begin{aligned}
& \mathcal{A}_{0}=\frac{V_{\bar{u}} T_{p_{1}} T_{p_{2}}}{4(2 \pi)^{d_{\bar{i}}}} \sqrt{\operatorname{det}\left(\Omega_{1} \Omega_{2}\right)} \operatorname{det}\left[\Gamma\left(\frac{U_{1}}{2 i \Omega_{1}}+1\right) \Gamma\left(\frac{U_{2}}{2 i \Omega_{2}}+1\right)\right] \\
& \times \frac{i(-1)^{\left(p_{1}+p_{2}\right) / 2} 2^{d_{\bar{u}}+1 / 2}}{\alpha^{\prime\left(p_{1}+p_{2}\right) / 2}} \frac{1}{\left(1+V_{1}^{2}\right)\left(1+V_{2}^{2}\right)} \\
& \times \int^{\infty} d t\left\{\left(\sqrt{\frac{\pi}{\alpha^{\prime} t}}\right)^{d_{\bar{i}_{n}}} \exp \left[-\frac{1}{4 \alpha^{\prime} t} \sum_{\bar{i}_{n}}\left(y_{1}^{\bar{i}_{n}}-y_{2}{ }^{\bar{i}_{n}}\right)^{2}\right] \prod_{\bar{i}_{c}} \Theta_{3}\left(\frac{y_{1}^{\bar{i}_{c}}-y_{2}^{\bar{i}_{c}}}{2 \pi R_{\bar{i}_{c}}} \mid \frac{i \alpha^{\prime} t}{\pi\left(R_{\bar{i}_{c}}\right)^{2}}\right)\right. \\
& \left.\times \lim _{t \rightarrow \infty}\left[\frac{e^{4 t}}{t^{1+\left(p_{1}+p_{2}\right) / 2}}+\frac{\operatorname{Tr}\left(\mathcal{S}_{(1) 1} \mathcal{S}_{(1) 2}^{T}\right)-2}{t^{1+\left(p_{1}+p_{2}\right) / 2}}\right]\right\} .
\end{aligned}
$$

The divergent part in the last bracket corresponds to the tachyonic closed string state. It differs from the same part in the papers by the coefficient $1 / t^{1+\left(p_{1}+p_{2}\right) / 2}$ which slows down this divergence. The other term of the last bracket is related to the contribution of the massless fields which goes to zero fast in the limit of large distances. It is notable that this damping factor just depends on the two D-branes dimensions not their relative configuration. So in the presence of the tachyon field the behavior of the interaction amplitude has changed in such a manner that in large distances the contribution of the graviton, dilaton and Kalb-Ramond fields disappears. This effect may be understood as follows.

According to the first equation of (17) the energy $p^{0}$ defines a linear potential, acting on the closed string. This potential completely originates from the background tachyon. This potential slows down the closed string motion, which happens for all closed string states, 
including the massless states. Therefore, the exchanged closed strings will cease and hence there is no long range force.

Vanishing of the D-branes interaction after long enough time also may be interpreted as rolling of the tachyon field in this limit [19]. Presence of the open string tachyon field implicates the instability of the D-brane. That is, in long time limit the tachyon rolls down to its minimum potential and hence the D-brane decays to closed string states and finally disappears. Thus, after long time there are no D-branes to interact with each other.

\section{Conclusions}

We obtained the boundary state of a closed string, emitted (absorbed) from (by) a moving brane in the presence of the background fields $B_{\mu \nu}$, tachyon and internal $U(1)$ gauge field. By partially compactifying the spacetime on tori, the formalism was applied to both compact and non-compact spacetime. We observed that the closed string can not wrap around the compact directions of the spacetime which are perpendicular to the brane's worldvolume. In addition, for a special tachyon matrix, the tachyon prevents the closed string from winding around the compact directions parallel to the brane's volume.

The interaction amplitude of two D-branes with arbitrary dimensions was calculated. The D-branes are parallel or perpendicular to each other. Due to the tachyon field, the interaction strength between the branes depends on all mode numbers of the exchanged closed string. The background fields, specially the tachyon field, affect the interaction. In other words, the background fields define an effective tension for each D-brane. In the corresponding potential, related to the interaction amplitude, the product of these effective tensions define a coupling constant. The value of this coupling constant indicates the strength of the interaction. Therefore, by adjusting the parameters $V_{1}, V_{2},\left\{U_{\mu \nu}^{(1)}, U_{\mu \nu}^{(2)}\right\},\left\{\mathcal{F}_{\mu \nu}^{(1)}, \mathcal{F}_{\mu \nu}^{(2)}\right\}$ and $\left\{R^{\mu} \mid \mu \neq 0\right\}$ we can control this strength.

In the large distance of the branes, the contribution of the massless states (i.e. graviton, dilaton and Kalb-Ramond fields) goes to zero and the divergence part related to the tachyonic closed string state considerably slows down. This can be understood by the decelerating potential which acts on the exchanging closed strings, and also by rolling of the tachyon which leads to the instability of the D-branes and hence decaying of them.

Although in this article we are dealing only with bosonic string, it is worth noting that similar consideration, concerning the effects of compactification, works for the superstring case since these effects are independent of the fermions. However, adding fermionic degrees of freedom to the present formalism is in progress. 


\section{References}

[1] J. Polchinski, Phys. Rev. Lett. 75, 4724 (1995).

[2] C. V. Johnson, "D-Branes", Cambridge University Press, 2003.

[3] P. Di Vecchia and A. Liccardo, "branes in string theory I", NATO Adv. Study Inst. Ser. C. Math. Phys. Sci. 556 (2000) 1-59.

[4] C.G. Callan, C. Lovelace, C.R. Nappi and S.A. Yost, Nucl. Phys. B288 (1987) 525; Nucl. Phys. B293 (1987) 83; Nucl. Phys. B308 (1988) 221.

[5] M. Billo, P. Di Vecchia and D. Cangemi, Phys. Lett. B400 (1997) 63.

[6] G. Lifstchytz, Phys. Lett. B388 (1996) 720.

[7] P. Di Vecchia, M. Frau, I. Pesando, S. Sciuto, A. Lerda and R. Russo, Nucl. Phys. B507 (1997) 259.

[8] M.M. Sheikh-Jabbari, Phys. Lett. B425 (1998) 48.

[9] S. Gukov, I.R. Klebanov and A.M. Polyakov, Phys. Lett. B423 (1998) 64.

[10] T. Kitao, N. Ohta and J. Ge Zho, Phys. Lett. B428 (1998) 68.

[11] D. Kamani, Mod. Phys. Lett. A15 (2000) 1655-1664, hep-th/9910043.

[12] H. Arfaei and D. Kamani, Phys. Lett. B452 (1999) 54, hep-th/9909167; Nucl. Phys. B561 (1999) 57, hep-th/9911146.

[13] D. Kamani and N. Nowrouzi, Braz. J. Phys. 41 (2011) 44-49, arXiv: 1012.5476 [hep-th].

[14] T. Lee, Phys. Rev. D64 (2001) 106004.

[15] A. Sen, JHEP 0405 (2004) 076.

[16] E. T. Akhmedov, M. Laidlaw and G. W. Semenoff, JETP Lett. 77: 1-6, 2003; PismaZh. Eksp. Teor. Fiz. 77: 3-8, 2003; Z. Rezaei and D. Kamani, "Moving Branes in Presence of the Background Tachyon Fields", J. Exp. Theor. Phys. 140 (2011), arXiv: 1106.2097 [hep-th].

[17] C.G. Callan and I.R. Klebanov, Nucl. Phys. B465 (1996) 473. 
[18] A. Sen, Int. J. Mod. Phys. A20 (2005) 5513; Int. J. Mod. Phys. A14 (1999) 4061.

[19] A. Sen, JHEP 0204 (2002) 048.

[20] M. Green, J. Schwarz and E. Witten, "Superstring theory", Vols. I and II (Cambridge University Press, 1987). 